\title{
A Dextral Primary Progressive Aphasia Patient with Right Dominant Hypometabolism and Tau Accumulation and Left Dominant Amyloid Accumulation
}

\author{
Young Kyoung Jang ${ }^{a, b}$ Seongbeom Park ${ }^{a, b}$ Hee Jin Kim ${ }^{a, b}$ \\ Hanna Cho ${ }^{c}$ Chul Hyoung Lyoo ${ }^{c}$ Sang Won Seo ${ }^{a, b}$ d Duk L. Na a, b, e \\ ${ }^{a}$ Department of Neurology and ${ }^{b}$ Neuroscience Center, Samsung Medical Center, \\ Sungkyunkwan University School of Medicine, 'Department of Neurology, \\ Gangnam Severance Hospital, Yonsei University College of Medicine, and Departments of

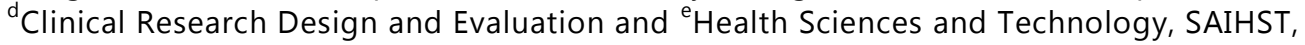 \\ Sungkyunkwan University, Seoul, Korea
}

\section{Key Words}

Primary progressive aphasia - Crossed aphasia - Asymmetric amyloid accumulation . Asymmetric tau accumulation

\begin{abstract}
Background: Primary progressive aphasia (PPA) is a degenerative disease that presents as progressive decline of language ability with preservation of other cognitive functions in the early stages. Three subtypes of PPA are known: progressive nonfluent aphasia, semantic dementia, and logopenic aphasia (LPA). Patients and Methods: We report the case of a 77year-old patient with PPA whose clinical findings did not correspond to the three subtypes but mainly fit LPA. Unlike other LPA patients, however, this patient showed a right hemisphere predominant glucose hypometabolism and tau accumulation and a left hemisphere predominant amyloid deposition. The right-handed patient presented with comprehension difficulty followed by problems naming familiar objects. This isolated language problem had deteriorated rapidly for 2 years, followed by memory difficulties and impairment of daily activities. Using a Korean version of the Western Aphasia Battery, aphasia was consistent with a severe form of Wernicke's aphasia. According to the brain magnetic resonance imaging and ${ }^{18} \mathrm{~F}$-fludeoxyglucose positron emission tomography results, right hemisphere atrophy
\end{abstract}

\section{KARGER}

Duk L. Na, PhD

Department of Neurology, Samsung Medical Center

Sungkyunkwan University School of Medicine

81 Irwon-ro, Kangnam-ku, Seoul, 06351 (Korea)

E-Maildukna@naver.com 
Jang et al.: A Dextral Primary Progressive Aphasia Patient with Right Dominant Hypometabolism and Tau Accumulation and Left Dominant Amyloid Accumulation

and hypometabolism, more predominant on the right hemisphere than the left, were apparent despite the fact that Edinburgh Handedness Questionnaire scores indicated strong righthandedness. On Pittsburgh compound B-PET, amyloid accumulation was asymmetrical with the left hemisphere being more predominant than the right, whereas ${ }^{18} \mathrm{~F}$-T807-PET showed a right dominant tau accumulation. Conclusions: This is the first report of atypical PPA, in which the patient exhibited crossed aphasia and asymmetrical amyloid accumulation.

(C) 2016 The Author(s)

Published by S. Karger AG, Basel

\section{Introduction}

Primary progressive aphasia (PPA) is a syndrome that presents as progressive decline of language ability while preserving other cognitive functions for at least 2 years [1]. PPA can be classified into three variants by language phenotypes [2]. The first type is progressive nonfluent aphasia (PNFA) characterized by nonfluent, labored speech and agrammatism without comprehension disability [1]. On brain imaging, atrophy and hypometabolism are usually seen in the left inferior frontal gyrus and anterior insula [2]. Pathologically, PNFA is mostly likely regarded as tauopathy [1]. The second type is semantic dementia (SD). Together with PNFA, SD is a language variant of frontotemporal lobar degeneration. The hallmark symptoms of SD are loss of word and object meanings and associated agnosia with preserved fluent speech [1]. SD is associated with atrophy and hypometabolism in the left anterior temporal area [2]. The underlying pathology of SD is heterogeneous, with TDP-type C being the most predominant [1]. The third type is logopenic aphasia (LPA), initially manifesting as anomic or conduction aphasia. Word-finding difficulty and impaired ability in sentence repetition are associated with left temporoparietal atrophy and hypometabolism [2]. LPA patients usually have the same pathology as Alzheimer's disease dementia (AD) [2].

We report a patient with PPA whose manifestation was difficult to classify into one of the three existing PPA subtypes. In addition, despite being strongly right-handed, the patient exhibited right hemisphere dominant glucose hypometabolism and left hemisphere dominant amyloid accumulation.

\section{Case Presentation}

A 77-year-old Korean man who was a retired office worker was brought to our memory disorder clinic by his wife, who reported that the patient's main problem over the past 2 years was difficulty understanding what other persons said. At first, the caregivers thought the man had a hearing problem. Six months after symptom onset, after a hearing aid prescribed by a local otolaryngology clinic did not correct the problem, the caregivers brought the patient to a local neurology clinic where he underwent brain magnetic resonance imaging (MRI). MRI showed bilateral periventricular white matter hyperintensities that were slightly more severe on the right side than the left and multiple stenoses in the anterior, middle and posterior cerebral arteries.

Caregivers recalled that at 3-4 months after onset, the patient started to have problems naming familiar, common, daily objects in addition to difficulty understanding speech. One year after onset, when asked questions, most of the patient's answers were unrelated to the question. However, the man was still able to read newspapers and use the Internet. Over the next year, his comprehension ability declined rapidly until he barely comprehended speech 
Jang et al.: A Dextral Primary Progressive Aphasia Patient with Right Dominant Hypometabolism and Tau Accumulation and Left Dominant Amyloid Accumulation

and never provided relevant answers to questions. At the same time, his memory was slightly impaired, for example, he could not recall a door-lock password. The patient started experiencing difficulty using public transit and taking medicines on his own. However, no obvious changes were noted in his behavior. When his family brought him to our clinic 2 years after symptom onset, he was moving slowly with a mildly shortened step.

The patient's past medical history was remarkable for hypertension, diabetes mellitus, hypothyroidism, and benign prostatic hyperplasia, but not for any history of developmental delay or disorder. The patient had received 16 years of education. Due to his severe language impairment, he could not perform actual motor skill tests to identify the dominant hand such as the Purdue Pegboard Test. However, the Edinburgh Handedness Questionnaire [3] revealed that he was strongly right-handed, on which he scored right-handed for all 10 questions. Family history showed that his mother had a stroke, while there was no family history of left-handedness.

A neurological examination showed that the patient was alert and able to utter sentences of 4-5 words and sounded fluent without being dysarthric or halting. However, his spontaneous speech often contained phonemic paraphasia or some jargon, making it difficult to understand what he was trying to say. The patient also showed severe auditory comprehension difficulty: for instance, when asked about his chief complaint, he asked 'what?' several times. The patient scored 0 of 30 on the Korean Mini-Mental State Examination because of language problems and cognitive impairment. His gait was slow with mild short steps. Cranial nerve functions, muscle volume, tone, power, and sensory functions were normal. Deep tendon reflexes were normal with no pathological reflexes.

Routine blood labs including serum vitamin $B_{12}$, syphilitic serological marker, creatinine, and thyroid-stimulating hormone showed no abnormalities. Apolipoprotein E genotype was $\varepsilon 4 / \varepsilon 4$. MRI showed bilateral periventricular white matter hyperintensities and diffuse brain atrophy on fluid-attenuated inversion recovery (FLAIR) sequence (fig. 1a). The patient had slightly more severe atrophy in the right temporal area than the left. In order to quantify the patient's cortical atrophy, we registered the patient's MR images into the MNI152 standard space using an affine linear transformation. Then, images were classified to white matter and gray matter using an artificial neural net classifier. The surfaces of the inner and outer cortices were restructured using 40,962 vertex points for each hemisphere automatically. Then, cortical thickness was computed applying the Euclidean distance between the linked vertex points of inner and outer surfaces. Subsequently, we measured W-scores based on the data collected from 55 normal elderlies (mean age: $68.8 \pm 3.2$, female sex ratio: 70.9\%). $\mathrm{W}$-score is the Z-score using the following formula with adjusting specific covariates: [(patient's raw value) - (value expected in the controls considering covariates)]/SD of the residuals in controls. Last, extracted values were reversed in order that positive numbers represent atrophic changes. The following special covariates were used: age, sex, education, and ICV. This patient showed decreased cortical thickness in all cortical area, and the right anterior temporal and parietal cortices were observed to be the thinnest regions (fig. 1b). ${ }^{18} \mathrm{~F}$-fludeoxyglucose positron emission tomography (FDG-PET) showed moderate hypometabolism in the bilateral fronto-parieto-temporal cortex (fig. 1c). Quantitative analysis of FDG-PET was done after PET images were coregistered to T1-weighted MRI. The quantitative regional values were measured by an automated volume of interest (VOI) analysis tool using the automated anatomical labeling atlas. SPM version 8 through Matlab 2014 (Mathworks, Natick, Mass., USA) was used for this analysis. To measure regional glucose metabolism, we used the standardized uptake value ratios (SUVRs) which is the cortical to reference region uptake ratio. Pons mean activity was set as a reference. We selected 28 cortical VOIs from both hemispheres. The selected cortical VOIs were made up of the following cortices: bilateral 
frontal (superior and middle frontal gyri, medial part of superior frontal gyrus, opercular part of inferior frontal gyrus, triangular part of inferior frontal gyrus, supplementary motor area, orbital part of superior, middle, and inferior orbital frontal gyri, rectus and olfactory cortex), posterior cingulate gyri, parietal (superior and inferior parietal, supramarginal and angular gyri, and precuneus), lateral temporal (superior, middle and inferior temporal gyri, and Heschl's gyri), and occipital (superior, middle, and inferior occipital gyri, cuneus, calcarine fissure, and lingual and fusiform gyri). The patient's FDG-PET SUVRs of the right hemisphere were lower than those of the left (left vs. right SUVR: frontal cortex: 1.46 vs. 1.35; temporal cortex: 1.32 vs. 1.09 ; parietal cortex: 1.42 vs. 1.21 ; occipital cortex: 1.58 vs. 1.48 ) (fig. 1d).

One month after the first visit to our clinic, the Korean version of the Western Aphasia Battery (K-WAB) was performed to identify the patient's aphasia type. Throughout the test, he had difficulty concentrating on the test and kept on speaking what he wanted to say. On a spontaneous speech test, the patient spoke sentences using 4-5 words. He sometimes understood a few words of questions and repeated those words, but his speech contents were not related to the conversation. On seven questions to elicit spontaneous speech, he gave only a single right answer to the question about his name. When asked to describe a picture, he formed sentences without agrammatism, but frequently used substitutes and jargons, resulting in a 2/10 score for informed content and 7.5/10 for fluency rating. The auditory comprehension part of the K-WAB consists of three subtests: yes-no questions (maximum score 60), auditory word recognition (maximum score 60), and sequential commands (maximum score 80). The patient's impairment on auditory comprehension was so severe that he could not understand even single words, let alone complex questions. Thus, he only scored $3 / 60$ in the yes-no subtest and scored none in other subtests. The patient also could not perform the repetition test and kept on speaking what he wanted to say (repetition: 0/10). When asked to name objects, the patient named 4 of 10 (object naming: 14/60). He was unable to perform tests about word fluency, sentence completion, and responsive speech. He also performed poorly at the reading $(0 / 10)$ and writing $(1.9 / 10)$ subtests. The overall results of the K-WAB were consistent with a severe degree of Wernicke's aphasia. The language disturbance precluded further neuropsychological tests.

At 3 years after symptom onset, the patient could not understand what others said and his utterance decreased drastically. At 3 years after onset, the patient underwent a Pittsburgh compound B (PiB)-PET which revealed amyloid accumulation mostly in the left fronto-parieto-temporal cortex (fig. 2a). PiB-PET was also analyzed using the same methods as FDG-PET except that for PiB-PET, the cerebellar gray matter was used as the reference area. Higher SUVRs were extracted in the right hemisphere than the left (left vs. right SUVR: frontal cortex: 2.01 vs. 1.75 ; temporal cortex: 1.82 vs. 1.57; parietal cortex: 2.07 vs. 1.76 ; occipital cortex: 1.68 vs. 1.50 ) (fig. 2 b). ${ }^{18 F-T 807-P E T ~ p e r f o r m e d ~ a t ~} 3.5$ years after symptom onset showed that tau deposition was more predominant in the right fronto-parietotemporal cortex than the left (fig. 3a). When SUVRs were calculated using the same process as PiB-PET, higher SUVRs were observed in the right cortices than the left (left vs. right SUVR: frontal cortex: 1.76 vs. 2.33; temporal cortex: 2.18 vs. 2.67; parietal cortex: 2.01 vs. 2.51; occipital cortex: 1.84 vs. 1.97) (fig. 3b).

\section{Discussion}

The patient in this study initially exhibited only language problems followed about 2 years later by other symptoms such as memory impairment. His slow movement and short 
step gait could be explained by white matter hyperintensities that might have been due to hypertension, diabetes mellitus, or multifocal intracranial cerebral artery stenosis. Therefore, this patient's symptoms, which were characterized by significant language deficit without other cognitive or behavioral changes at the earlier stages, were compatible with PPA.

Our patient's manifestation was not archetypal and did not fit into the three existing PPA subtypes. After excluding the possibility of PNFA, the patient's symptoms alone were mostly consistent with SD because he presented with difficulty in comprehension and naming familiar objects. However, he scored better on a confrontational naming test than on other aphasia subtests and showed only phonemic paraphasic errors, not semantic paraphasic errors. Furthermore, his MRI findings did not indicate SD. Given his amyloid positivity, the most likely diagnosis for this patient was LPA. However, comprehension ability was strikingly impaired compared to other symptoms and a detailed history revealed that comprehension difficulty preceded word retrieval deficit, which is not typical of LPA.

We identified two unique findings for this patient. The first is crossed aphasia. The patient was strongly dextral both by history and the Edinburgh Handedness Questionnaire. However, glucose hypometabolism was more significant in the right cortex than the left cortex on FDG-PET. The right temporal area also showed more severe atrophy than the left area on MRI. To our knowledge, this is a rare phenomenon in PPA. Four cases of crossed aphasia in both PNFA [4-6] and LPA [7-10] have been reported, with no report in SD.

The second unique finding was asymmetrical amyloid accumulation on PiB-PET. Reports of about 77 patients with LPA who underwent amyloid PET found that $87.0 \%(67 / 77)$ were amyloid positive [2, 11-14]. Interestingly, all showed bilateral symmetric amyloid accumulation similar to AD, despite marked language symptoms. Similarly, other AD variants such as early onset $\mathrm{AD}$ and posterior cortical atrophy showed diffuse amyloid distribution. Thus, the general pattern of amyloid accumulation that was observed through PET does not seem to match the unique clinical symptoms [12]. However, one recent autopsy study reported that 28 of 31 (90\%) PPA patients showed preponderant neurodegenerative pathologies including amyloid, tau, and TDP in the language dominant hemisphere [15]. This result may be consistent with $18 \mathrm{~F}-\mathrm{T} 807-\mathrm{PET}$ findings of our patient.

Our study has a limitation as the first evaluation was not made at the initial stage. The patient visited our clinic 2 years after onset and his language impairment at the first visit was already too advanced for detailed tests.

In conclusion, we report a case of PPA with asymmetrical amyloid accumulation and crossed aphasia. Future studies about possible pathologies with more cases are needed.

\section{Acknowledgement}

This study was supported by a grant of the Korean Health Technology R and D Project, Ministry of Health and Welfare, Republic of Korea (HI14C2746).

\section{Statement of Ethics}

The authors have no ethical conflicts to disclose. 


\section{Case Reports in Neurology}

\section{Disclosure Statement}

\author{
The authors have no conflicts of interest to disclose. \\ The authors have no conflicts of interest to disclose.
}

Jang et al.: A Dextral Primary Progressive Aphasia Patient with Right Dominant

Hypometabolism and Tau Accumulation and Left Dominant Amyloid Accumulation

\section{References}

1 Bang J, Spina S, Miller B: Frontotemporal dementia. Lancet 2015;386:1672-1682.

-2 Rabinovici GD, Jagust WJ, Furst AJ, Ogar JM, Racine CA, Mormino EC, et al: Abeta amyloid and glucose metabolism in three variants of primary progressive aphasia. Ann Neurol 2008;64:388-401.

3 Oldfield RC: The assessment and analysis of handedness: the Edinburgh inventory. Neuropsychologia 1971;9:97-113.

4 Repetto C, Manenti R, Cotelli M, Calabria M, Zanetti O, Borroni B, et al: Right hemisphere involvement in non-fluent primary progressive aphasia. Behav Neurol 2007;18:239-243.

5 Jeong EH, Lee YJ, Kwon M, Kim JS, Na DL, Lee JH: Agrammatic primary progressive aphasia in two dextral patients with right hemispheric involvement. Neurocase 2014;20:46-52.

-6 Spinelli EG, Caso F, Agosta F, Gambina G, Magnani G, Canu E, et al: A multimodal neuroimaging study of a case of crossed nonfluent/agrammatic primary progressive aphasia. J Neurol 2015;262:2336-2345.

7 Parente A, Giovagnoli AR: Crossed aphasia and preserved visuospatial functions in logopenic variant primary progressive aphasia. J Neurol 2015;262:216-218.

-8 Mesulam M, Weintraub S, Parrish T, Gitelman D: Primary progressive aphasia: reversed asymmetry of atrophy and right hemisphere language dominance. Neurology 2005;64:556-557.

-9 Demirtas-Tatlidede A, Gurvit H, Oktem-Tanor 0, Emre M: Crossed aphasia in a dextral patient with logopenic/phonological variant of primary progressive aphasia. Alzheimer Dis Assoc Disord 2012;26:282284.

10 San Pedro EC, Deutsch G, Liu HG, Mountz JM: Frontotemporal decreases in rCBF correlate with degree of dysnomia in primary progressive aphasia. J Nucl Med 2000;41:228-233.

-11 Leyton CE, Villemagne VL, Savage S, Pike KE, Ballard KJ, Piguet O, et al: Subtypes of progressive aphasia: application of the International Consensus Criteria and validation using beta-amyloid imaging. Brain 2011;134:3030-3043.

12 Lehmann M, Ghosh PM, Madison C, Laforce R Jr, Corbetta-Rastelli C, Weiner MW, et al: Diverging patterns of amyloid deposition and hypometabolism in clinical variants of probable Alzheimer's disease. Brain 2013;136:844-858.

13 Whitwell JL, Lowe VJ, Duffy JR, Strand EA, Machulda MM, Kantarci K, et al: Elevated occipital beta-amyloid deposition is associated with widespread cognitive impairment in logopenic progressive aphasia. J Neurol Neurosurg Psychiatry 2013;84:1357-1364.

14 Ikeda M, Tashiro Y, Takai E, Kurose S, Fugami N, Tsuda K, et al: CSF levels of Abeta1-38/Abeta140/Abeta1-42 and (11)C PiB-PET studies in three clinical variants of primary progressive aphasia and Alzheimer's disease. Amyloid 2014;21:238-245.

15 Mesulam M, Weintraub S, Rogalski EJ, Wieneke C, Geula C, Bigio EH: Asymmetry and heterogeneity of Alzheimer and frontotemporal pathology in primary progressive aphasia. Brain 2014;137:1176-1192. 


\section{Case Reports in Neurology}

\begin{tabular}{l|l}
\hline Case Rep Neurol 2016;8:78-86 \\
\hline DOI: 10.1159/000445538 & $\begin{array}{l}\text { ○ 2016 The Author(s). Published by S. Karger AG, Basel } \\
\text { www.karger.com/crn }\end{array}$ \\
\hline
\end{tabular}

Jang et al.: A Dextral Primary Progressive Aphasia Patient with Right Dominant Hypometabolism and Tau Accumulation and Left Dominant Amyloid Accumulation

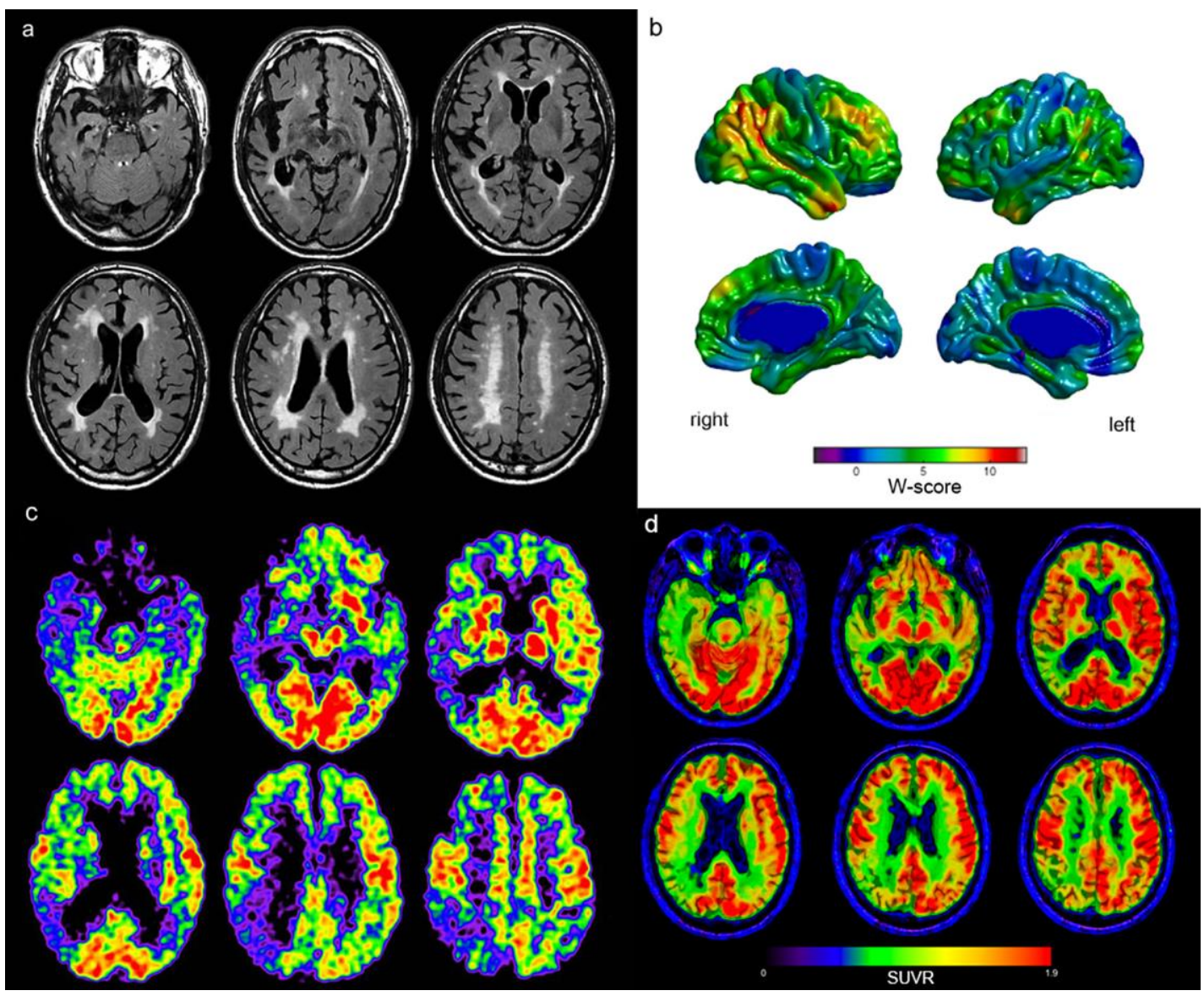

Fig. 1. a FLAIR images show diffuse atrophy and periventricular white matter hyperintensities that are probably due to hypertension, diabetes mellitus, or multifocal intracranial cerebral artery stenosis in this patient. Temporal atrophy appears to be more severe in the right than the left hemisphere. b Cortical thickness analysis of MRI. Compared to data collected from 55 normal elderlies, the patient's MRI shows that atrophy was mostly noticeable in the right anterior temporal and parietal area. c FDG-PET images show moderate hypometabolism in the bilateral fronto-parieto-temporal cortex, more remarkable in the right than the left hemisphere. $\mathbf{d}$ FDG-PET SUVR. Glucose hypometabolism is more severe in the right than the left fronto-parieto-temporal cortices compared to the mean activity of the pons area (left vs. right SUVR: frontal cortex: 1.46 vs. 1.35 ; temporal cortex: 1.32 vs. 1.09 ; parietal cortex: 1.42 vs. 1.21 ; occipital cortex: 1.58 vs. 1.48$)$. 
Jang et al.: A Dextral Primary Progressive Aphasia Patient with Right Dominant Hypometabolism and Tau Accumulation and Left Dominant Amyloid Accumulation

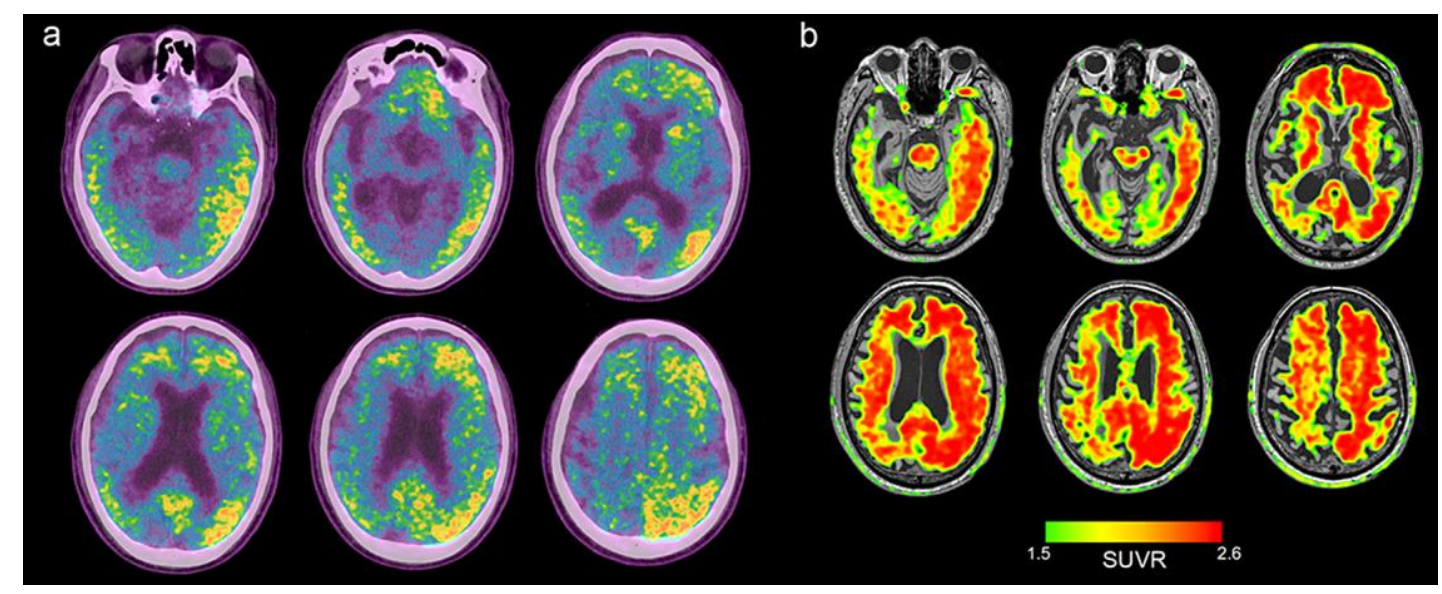

Fig. 2. a PiB-PET images show asymmetrical amyloid accumulation which is more outstanding in the left hemisphere than the right. b PiB-PET SUVR. Compared to cerebellar gray matter, amyloid accumulation is dominant in the left fronto-parieto-temporal cortices (left vs. right SUVR: frontal cortex: 2.01 vs. 1.75; temporal cortex: 1.82 vs. 1.57 ; parietal cortex: 2.07 vs. 1.76 ; occipital cortex: 1.68 vs. 1.50 ). 
Jang et al.: A Dextral Primary Progressive Aphasia Patient with Right Dominant Hypometabolism and Tau Accumulation and Left Dominant Amyloid Accumulation

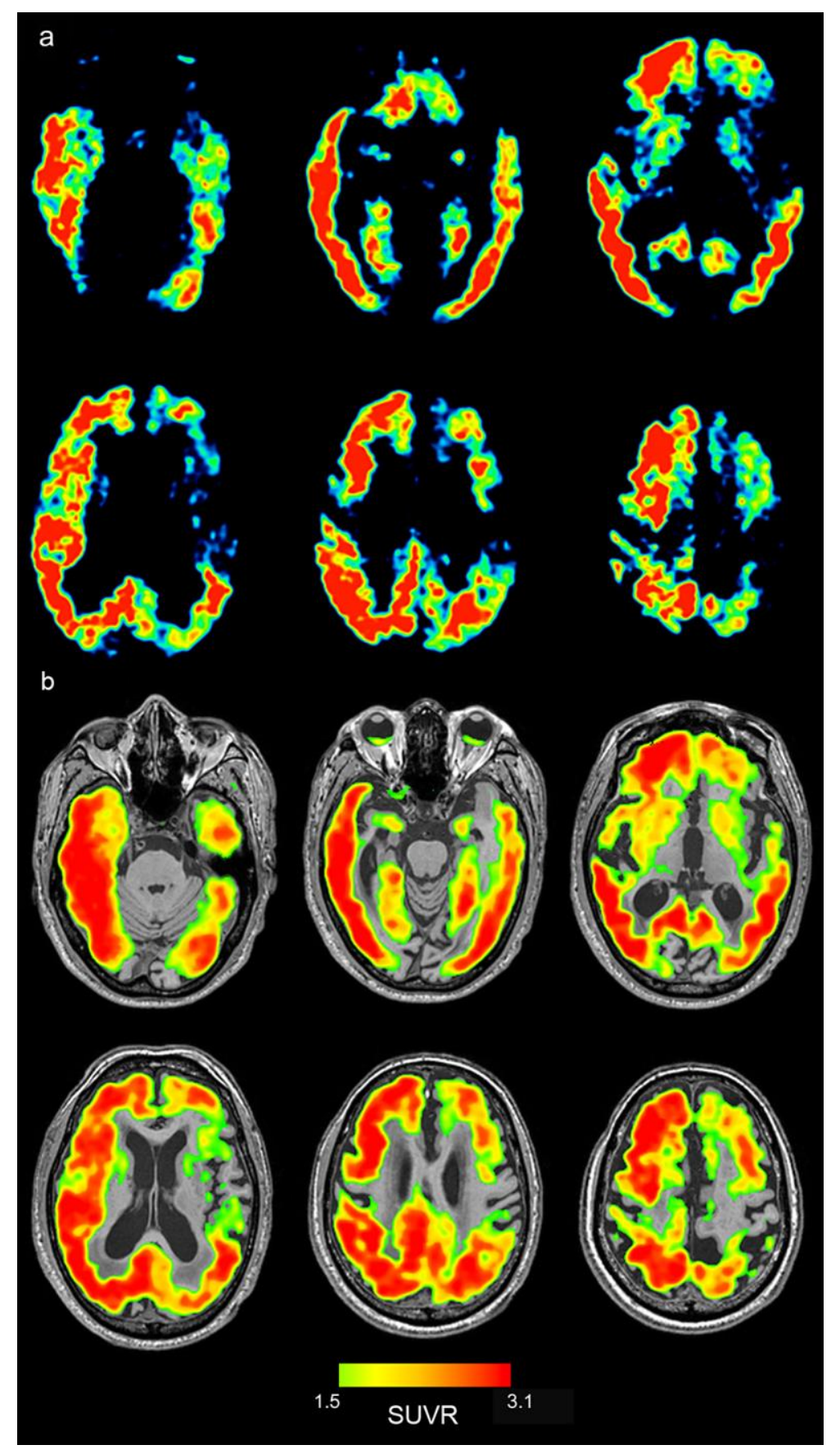

Fig. 3. a ${ }^{18 F-T 807-P E T ~ i m a g e s ~ s h o w ~ a n ~ a s y m m e t r i c ~ t a u ~ a c c u m u l a t i o n ~ w h i c h ~ i s ~ m o r e ~ p r o m i n e n t ~ i n ~ t h e ~}$ right than the left hemisphere. b ${ }^{18 F-T 807-P E T ~ S U V R . ~ C o m p a r e d ~ t o ~ c e r e b e l l a r ~ g r a y ~ m a t t e r, ~ t a u ~ a c c u m u l a-~}$ tion is more prominent in the right fronto-parieto-temporal cortices than the left (left vs. right SUVR: frontal cortex: 1.76 vs. 2.33; temporal cortex: 2.18 vs. 2.67; parietal cortex: 2.01 vs. 2.51; occipital cortex: 1.84 vs. 1.97). 